\title{
The Role and Application of Decorative Materials in Interior Design
}

\author{
Jing Zeng, Mengyang Jiang \\ College of Forestry, Sichuan Agricultural University, Wenjiang 611130, China
}

Keywords: decorative materials; interior design; role; innovative applications

\begin{abstract}
Modern decoration materials are divided into two parts: outdoor decoration materials and interior decoration materials. The categories and applications of decorative materials are different because of the different indoor and outdoor environment, function and demand. This article mainly emphasizes the role and application of interior decoration materials. In interior design, designers must fully understand the status of decoration materials in interior design, function orientation, artistic characteristics and development prospects, and then, the use of decorative materials can be more efficient, to meet user demand for the basic function of indoor environment, and also to meet the yearning and pursuit for a better life of people in present stage.
\end{abstract}

\section{Introduction}

The material is the representation of the entity, and the material as the basic substance which forms the building body is the carrier of the expression of architectural form and the object of the most direct perception of the experiencers. Each kind of material has its unique design vocabulary, which contains local climate and cultural characteristics. The texture, color and hardness of different materials can bring different feelings to the experiencers. The form processing of materials is an inherent part of architectural design. The form is the final presentation of architecture, and the architecture carries a lot of cultural meaning and spiritual connotation in the form of the entity. ${ }^{[1]}$ Interior decoration material is the material basis of interior design. Interior decoration materials can not only improve the art of indoor environment, make people get beautiful enjoyment, but also with the green environmental protection, multi-functional, comfortable, safe and reliable, and so on, play a protection main body structure, service life extension, and satisfy some special requirements, is indispensable to modern building decoration materials. Full understanding of its role and innovative application can not only bring efficiency and inspiration to designers, but also promote the development of human social science and technology

\section{The role of decorative materials in interior design}

In the modern interior design, we must consider the factors such as color, shape, material, function, safety and environmental protection of interior decoration, which are mostly determined by the choice of interior decoration materials. Decoration materials plays an important role in interior design and decoration of the new material not only meet the aesthetic needs of the user in the form at the same time, modern interior design trends, through the mutual collocation between different materials to create new artistic features, thus forming a new era decoration style. With the development of science and technology, the development of new materials change rapidly, continuously improve people's living standard, so the demand for high quality of life is also growing, green environmental protection has gradually become the mainstream of modern interior design, the requirements of the quality and the function of decoration materials must meet the green environmental protection standards, researchers must constantly improve the lack of decoration materials, research and create new decorative materials, and long-term protection of the health of society, and promote the sustainable development of society.

\subsection{The important status of decorative materials in interior design}

The contents of interior design include indoor space organization and interface processing, 
indoor lighting, color design and material selection, indoor furnishings (furniture, lamps and lanterns, Lv Zhi) design and selection. These all interact with and interact with the interior decoration materials. The relationship between spatial organization and spatial hierarchy processing is often through the design of indoor space shape, the use of indoor space wall, ground, top surface of different materials, furnishings to reflect the object background, materials required for interior space design, but also to provide important inspiration for interior space design, the two are interdependent and mutual development. Treatment of interior space must utilize existing decorative materials, and new decoration materials continue to be demand, which promote the development of materials and technology, the value of the material itself is not high, only when the material is used in some special requirements in order to reflect its highest value.

Indoor interface processing refers to the bottom round into interior space (wall), ground (floor), the side and top surface (ceiling, ceiling) from all walks of life the use function and the analysis of the characteristics, such as decorative materials including indoor interface materials.

Indoor lighting refers to the natural lighting and artificial lighting of indoor environment. Lighting can not only satisfy lighting and lighting requirements of normal working and living environment, but also play an important role in lighting indoor environment and atmosphere. Light through different materials will form the refraction and reflection effects, thus forming different indoor lighting effects, such as in the KTV room, the walls often use mirrors or glass decorative materials, when the room lights open after exposure to the wall, a reflection of different lighting effects to create KTV active atmosphere, at the same time, give people a wide visual effect of mirror material can be reduced, the original narrow sense of space is boring.

Color is the most vivid and active factor in interior design, and indoor color often gives people the first impression of indoor environment. Color is the most expressive force. It forms rich associations, profound meanings and symbols through the physiological, psychological and physical effects produced by people's visual experience. The color of decoration materials can replace the traditional wall paint. Its unique texture can improve indoor environmental quality, such as TV background wall, art wall, etc., often using special decoration materials, creating indoor climax space, increasing the level sense and coordination of space.

Indoor furnishings are very prominent in practicality and appreciation. They are usually in a prominent position in vision. Furniture is also directly related to human body and feels the closest distance. Furniture, furnishings, lamps and greening, etc. play an important role in the indoor environment atmosphere and the style of interior design. Interior furnishings and interior decoration materials must be chosen according to the interior design style. In essence, interior decoration materials determine the trend of interior design style, so indoor furnishings must be matched with them to create an elegant indoor environment. Indoor greening has a special role that can not be replaced in modern interior design. Indoor greening has the function of improving indoor microclimate and absorbing dust. Indoor decoration materials are now tending to be green and environment-friendly, materials and greening, more can bring natural atmosphere to the indoor environment, pleasing to the eye.

Generally speaking, decorative materials are essential in interior design, and it is a very important position.

\subsection{Artistic features of decorative materials}

The artistic characteristics of decorative materials often directly stimulate the creative power of design, guide the fashion of design, and define the aesthetic characteristics, artistic conception, atmosphere and information transmission of interior space with its own artistic expression. Excavating the visual artistic features of the material and using it skillfully has become the new focus of the application of the material innovation in the interior design ${ }^{[2]}$. The artistic features of decorative materials including color, texture, texture and other specifications, combination of interior design cannot do without decoration material collocation, materials affect people on the indoor environment of visual and tactile sensations, and triggered a series of psychological emotion, form a rich imagination, profound meaning and symbol. Material's smoothness and roughness, 
softness and hardness, color and luster reflect its own characteristics. The combination of decorative materials can show a certain theme and create a specific atmosphere, making the space full of distinct personality and conveying different environmental information. The color, texture, texture and specifications of decorative materials have their own artistic characteristics. People capture and interpret these environmental information, and extract extensive characteristics from visual stimulation to form environmental perception ${ }^{[3]}$. Designers can classify and match according to the artistic characteristics of different decorative materials, so as to get a series of decorative materials, then allocate them in a certain proportion, and make rational use of them in an indoor space to form an overall artistic style.

\subsection{Functional positioning of decorative materials}

The function of decorative material is divided into two parts: decoration function and use function. Decorating function refers to the surface decoration of materials presented indoors, such as form, color, interface, texture, effect and so on. It sets off the whole indoor atmosphere, reflects the interior decoration style, and meets people's aesthetic needs. Use function refers to the needs of different users in different space environment and space decoration materials are different, so the decoration materials have different functions, such as the kitchen needs with fireproof function decoration materials; toilet needs with dampproof function decorative materials; KTV need to have the sound insulation function of decorative materials; summer needs with radiating function decoration materials; winter need with function of warm decoration materials...

The designer must according to the nature of the use of space, the environment, to determine the function of decoration materials demand space location, select the appropriate decoration materials, decoration materials to avoid excess function, renovation costs are too high, such as the use of sound insulation, moisture-proof decoration materials in the balcony, the position of the balcony was originally ventilated to the sun. It was not necessary and wasted the cost.

\subsection{Development prospect of decorative materials}

The development trend of new decorative materials is from low level to high level, from single function to multi-function, from the field production of artificial and chemical products to the standardization, finished products, and intelligent environmental protection materials. This is the inevitable product of the human development of high technology. With the improvement of people's living standard, science and technology is highly developed and people's demands are higher and higher. The lightweight, durable, good texture, decorative and intelligent environment-friendly decorative materials are becoming more and more popular in interior decoration ${ }^{[4]}$.

The development of decorative materials gradually tends to green and environmental protection materials and composite materials. The emergence of new decorative material to replace the old materials, the basis of quality, improve the functional diversity, reduce the complexity of technology, material saving renovation costs, meet people's different needs and to improve the indoor air quality, improve the quality of the living environment of people. The emergence of new materials is to make up for the shortage of old materials. So repeatedly, it promotes the continuous development and evolution of interior decoration materials, and brings better and healthier living environment to people.

\section{Innovative application of decorative materials in interior design}

Innovation refers to a process characterized by new thinking, new inventions and new descriptions. There are three meanings: updating, creating new things and changing ${ }^{[5]}$. The development of decorative materials is changing with each passing day. The emergence of new materials brings fresh inspiration to designers. People's minds are constantly innovating, interior design styles are gradually changing, people are willing to pursue new things, and the word "innovation" has been widely mentioned in modern life. In interior design, to make good use of good decorative function of all kinds of decorative materials and the use of function in the foundation to meet the basic decoration and function, to break the traditional mode of use, use of 
hyperbole, avant-garde decoration around the design concept of innovative thinking, to create a new decorative effect, leading interior design trends, into fun with the charm of the indoor space, to meet the personalized needs of people.

\subsection{The visual impact of decorative materials}

Gestalt psychology aesthetics principle that people's perception is not objective imitation, but each object perception activities dotting. Visual perception is the activity of visual design language, at the same time, it restricts the way of visual thinking, and uses the transformation of graphic symbols to stimulate creative thinking, emphasizing the resonance between the design objects and the whole in the mind. From the visual to the psychological process, give people a strong impact, awakening the unconscious mind, so as to achieve the purpose of transmitting information ${ }^{[6]}$. The interior decoration of a variety of materials, collocation of different materials to form different visual manifestation of each person from a different angle, thinking is different, which lead to the visual association is also different, innovative applications of decorative materials requires the designer to use the creative use of materials, to grasp the psychological characteristics and visual rules, grasp the commonness and individuality of people break the normal procedure. The visual association, so as to create out of the ordinary visual space, bring unexpected visual impact to the people.

\subsection{Humanistic emotional expression of interior design}

In the field of design, every piece of work has a rich cultural and emotional connotation. Emotion has always been the spirit and life of the works of art, and so is the design of the architectural space. Interior designers inject many feelings into architectural space, make people more harmonious with space, and let people experience more about the cultural atmosphere brought by materials. Architectural decoration material is the carrier of spatial emotion, it is perceived by the texture, texture, color and form of the material. How to use the performance and characteristics of the material to better show the meaning of the beauty of space is the designer's pursuit. In today's information society, many concepts have been subverted, many materials have been developed and utilized, and many of the traditions have been defined by new concepts. This requires designers to explore new materials and traditional materials for more meaning and use [6] with new thinking. Decoration materials is the foundation to build the style of interior design, interior design style often through which contain human emotions to reflect human emotions only with indoor space, can deeply impress people, arouse people's sympathy, can truly reflect the value of existence of interior design style.

\subsection{The aesthetic art of decorative materials}

Interior decoration design involves many factors, such as society, culture, economy, market, science and technology, and even politics. Its aesthetic features also change with the changes of many factors, and determine its aesthetic particularity, times, universality and pluralism. The in-depth and meticulous research of interior design is very necessary for design and aesthetics. It provides a broader direction for contemporary interior design and cultural research. Interior design is recreating in the space form given by the building. It has the function of decorating buildings and their environment, and it also has the expression characteristic of general plastic arts ${ }^{\text {[7] }}$. People from the basic functional requirements of the beginning of the progressive development of the aesthetic needs of human beings are different, that modern people on indoor environmental requirements continue to improve, it also requires the decoration materials must have the characteristics of beauty, and this is not limited to material properties, appearance, texture, but through cooperation between different decorative materials by means of new technology, show the characteristics of artistic beauty, meet the people's growing aesthetic ideas.

\subsection{Regenerated use and innovative design of discarded decorative materials}

The same material presents different artistic values in different historical stages, and the rational use of the same material can get different and meaningful interpretation. Waste materials, as long as 
they are used properly, play an important role in expressing design concepts, reflecting space interest and so on. To a certain extent, they can save the cost of decorative materials. It is an environmentally friendly design trend, [2], which is worth promoting.

The interior decoration material is the carrier of the expression of the space art design, and the material basis of the design creativity. In the full understanding of the performance based on the decoration materials, flexible collocation according to the use of the environment, reasonable choice, give full play to each kind of material strengths, to do their best, make the best use of materials, to meet the needs of interior space design. Artistic design and materials are complementary to each other, and any creative design needs to be achieved through material. Try to keep the external form of the original waste decoration materials, the dual function of practical and aesthetic needs to follow the design of products, low carbon concept in the light of its general trend of regeneration using waste decoration materials, using the laws of art scattered reconstruction design, make waste decoration materials again coruscate new vitality of the arts, art designers play subjective initiative the design of art decoration materials, abandoned in the furnishings of creative focus on the performance of ${ }^{[8]}$.

\section{Conclusion}

Decoration materials plays an important role in interior design, interior design is the basic medium of artistic creation and function requirements, make full use of existing materials, in-depth study of its defects and shortcomings, combined with the continuous improvement of environmental protection requirements, and create new materials, materials analysis categories based on new materials, select the appropriate the innovative application form, and combined with the recycling and other aspects of innovation, human visual manifestation of emotional expression, aesthetic art shows and abandoned decoration materials, efforts to develop new creative thinking, so as to meet the needs of more people, in ensuring a healthy indoor environment, indoor air quality based on the created with modern people's aesthetic and values, meet the personalized and comfortable indoor environment, and continuously meet the people's needs, along with science and technology Pace, continuous evolution.

\section{Acknowledgements}

Punds project: Key Laboratory of Wood Industry and Furniture Engineering of Sichuan Provincial Colleges and Universities

\section{References}

[1] Huang Jianjun. Analysis of the semiotics of materials [D]. Tianjin university,2011.

[2] Hu Jianzhong. An innovative application study of decorative materials in interior design [J]. Sichuan architectural science research,2013,39(06):293-296.

[3] Xu Leiqing. Ergonomics and environmental behavior [M]. Beijing: China construction industry press, 2006.

[4] Hu Xiao. The function of decorative materials in interior design and ecological environmental protection research [D]. Qingdao university of technology,2013.

[5] Zhang Ye. Aesthetic principles in visual design [J]. Knowledge economy,2012(20):123.

[6] Ge Ming. Value of materials in architecture and interior design [D]. Northeast normal university,2011.

[7] Zhang Bo. On the artistic style of interior decoration design [D]. Hebei university,2007.

[8] Yi Rui. Artistic regenerative application of abandoned decorative materials in modern soft decoration [J]. Hunan packaging,2012(01):33-35. 